\title{
Capacidade operacional e eficiência de campo da produção de silagem de milho cultivado em pivô central: estudo de caso
}

\author{
Luiz Henrique de Souza ${ }^{1 *}$, Victor Lucas Fernandes ${ }^{2}$
}

\begin{abstract}
Resumo
Para se realizar o gerenciamento agrícola, uma das formas mais utilizadas é a quantificação da Eficiência de Campo. Por meio desta técnica o produtor rural pode avaliar se o equipamento está sendo subutilizado. Este trabalho tem como objetivo avaliar a Capacidade Operacional e a Eficiência de Campo da produção de silagem de milho cultivado em pivô central: estudo de caso. Todas as operações foram realizadas utilizando-se um trator Massey Ferguson, modelo MF 291, 4x2 TDA, potência nominal de 73,6 kW (100cv) a 2200 rpm. Para avaliação da Capacidade Operacional foi realizada a medição do tempo total de trabalho de cada uma das operações separadamente desde o preparo do solo até a colheita em toda a área. A Eficiência foi determinada pela razão da Capacidade Operacional Efetiva pela Teórica. Os implementos que necessitaram de tempo para preparo e abastecimento de insumos, preparo de calda e troca de carreta foram os que apresentaram menor eficiência. Mesmo apresentando maior largura do que os outros implementos, o pulverizador apresentou menor eficiência devido ao maior tempo de deslocamento e abastecimento do equipamento. O tempo total de manobras alcançado pelos equipamentos devido à área circular do terreno pode ter contribuído para a baixa eficiência das operações.
\end{abstract}

Palavras-chave: Desempenho operacional. Máquinas agrícolas. Mecanização agrícola.

\section{Operational capacity and field efficiency of corn silage production cultivated in a central pivot: a case study}

\begin{abstract}
One of the most used ways to perform agricultural management is to quantify the field efficiency. The rural producer may assess whether the equipment is being underutilized through this determination. This work aims to evaluate the Operational Capacity and Field Efficiency of corn silage production cultivated in a central pivot: a case study in an area of 2.3 ha. All the operations were performed using a Massey Ferguson tractor model MF 291, 4x2 TDA, nominal power of $73.6 \mathrm{~kW}(100 \mathrm{hp})$ at $2200 \mathrm{rpm}$. In order to evaluate the Operational Capacity, the total working time of each operation was measured separately from the preparation of the soil to the harvest in the whole area. The Efficiency was determined by the ratio of the Effective Operational Capacity for the Theoretical. The implements that required time for preparation and supply of inputs, preparation of spray solution and cart exchange were the ones that showed less efficiency. Even with greater width than the other implements, the sprayer presented the least efficiency due to the longer time of equipment movement and supply. The total maneuver time achieved by the equipment due to the circular land area may have contributed to the operations' low efficiency.
\end{abstract}

Keywords: Operating performance. Agricultural machines. Agricultural mechanization.

\footnotetext{
${ }^{1}$ Universidade Federal de Minas Gerais. Instituto de Ciências Agrárias. Montes Claros, MG. Brasil. https://orcid.org/0000-0003-3939-4871.

${ }^{2}$ Universidade Federal de Minas Gerais. Instituto de Ciências Agrárias. Montes Claros, MG. Brasil.

https://orcid.org/0000-0002-2623-7087.

*Autor para correspondência: lhesouza@yahoo.com.br
}

Recebido para publicação em 07 de novembro de 2019. Aceito para publicação em 02 de fevereiro de 2020.

e-ISSN: 2447-6218 / ISSN: 2447-6218. Atribuição CC BY. 


\section{Introdução}

Para se realizar o gerenciamento agrícola, uma das formas mais utilizadas é a avaliação da eficiência de cada operação. Por meio desta determinação o produtor rural tem a condição de avaliar se o equipamento está sendo subutilizado.

Conforme Duarte Júnior et al. (2008), a maioria das operações agrícolas empregadas na instalação de uma cultura, desde o preparo do solo até colheita, pode ser mecanizada. Estas operações podem apresentar grande eficiência e retorno econômico ao produtor, desde que bem conduzidas, empregando tecnologia e maquinário adequado. Para isso, os autores salientam a importância do dimensionamento adequado do conjunto trator e máquina agrícola para proporcionar elevada capacidade operacional e a redução dos custos e do tempo de trabalho.

Baio et al. (2013) descrevem a escolha do equipamento adequado para uma propriedade agrícola como uma das etapas mais importantes do processo produtivo, e informam ainda que, a correta adequação da capacidade da máquina em relação à área é de extrema importância, pois afeta diretamente os custos produtivos.

Segundo Pequeno et al. (2012), o gerenciamento da produção agrícola deve minimizar o custo da maquinaria agrícola em relação ao custo total de produção. Nesse sentido, indicam a seleção do maquinário como um importante componente a ser considerado.

Silveira, Yanai e Kurachi (2006) informam que as máquinas selecionadas nas operações agrícolas devem ter capacidade satisfatória para concluir todas as operações dentro dos períodos críticos de tempo disponíveis e que com a utilização de máquinas cada vez maiores e mais caras, cada equipamento adquirido representa gastos também maiores. Salientam ainda que a capacidade de trabalho da máquina e a eficiência de campo, são fatores fundamentais no gerenciamento da utilização da maquinaria agrícola.

A Norma da ASAE D497.4 (1999) estipula um intervalo de eficiência para utilização dos diversos equipamentos agrícolas em suas respectivas velocidades e muitos autores utilizam estes valores. Vários trabalhos têm demonstrado melhor capacidade operacional com o aumento da velocidade de trabalho e largura dos implementos; porém o formato da área, declividade do terreno, tempo de paradas, distância de abastecimento e de descarga, são também fatores de extrema importância na avaliação da capacidade operacional e eficiência dos equipamentos.

Araldi et al. (2013) avaliando a eficiência operacional na colheita mecanizada em lavouras de arroz irrigado concluíram que as variáveis com maior correlação com a redução das eficiências, encontradas em seu experimento, foram tempo e velocidade de deslocamento, para eficiência de campo, e tempo de descarga, para eficiência de tempo. Concluíram ainda que as manobras e descargas consumiram em média $11,1 \%$ e $10,8 \%$ do tempo total nas operações analisadas, respectivamente.

Assim sendo, este trabalho tem por objetivo realizar um estudo de caso para avaliar a Capacidade Operacional e a Eficiência de Campo da produção de silagem de milho cultivado em pivô central.

\section{Materiais e métodos}

Os ensaios foram conduzidos em uma área irrigada sob pivô central de 2,3 ha cultivada com milho, localizada na fazenda experimental do Instituto de Ciências Agrárias da UFMG na cidade de Montes Claros- MG.

Todo o ciclo do milho desde o preparo do solo até a colheita foi monitorado para avaliar a eficiência operacional de todos os conjuntos mecanizados utilizados na produção de silagem.

O trator utilizado em todas as operações foi um Massey Ferguson, modelo MF 291, 4 x 2 TDA, com potência nominal no motor de 73,6 kW (100 cv) a 2200 rpm.

O solo foi preparado com uma grade aradora de 14 discos "off-set" marca Baldan com 1,8 m de largura, seguida de duas gradagens com grade destorroadora 28 discos "off set" Super Tatu com 2,37 m de largura.

O milho foi semeado com uma semeadora-adubadora Tatu Marchesan modelo PST PLUS com espaçamento de 0,8 m entre linhas composta por 3 unidades semeadoras, com largura total de $3 \mathrm{~m}$.

O cultivo químico foi realizado com a aplicação do herbicida Glifosato e o inseticida Klorpan utilizando-se um pulverizador de barras da Jacto com 19 bicos espaçados de $0,5 \mathrm{~m}$ com $9 \mathrm{~m}$ de largura total.

A área foi adubada com uma aplicação de Sulfato de Amônio e Cloreto de Potássio e duas aplicações com ureia utilizando-se, para ambos, um cultivador adubador de cobertura, sem as hastes, da Tatu Marchesan modelo CAC dotado de 4 linhas com 2,80 m de largura.

O processamento do milho para silagem foi realizado com uma colhedora de uma linha da Nogueira Pecus 9004 na rotação nominal de 540 rpm na TDP do Trator.

Todas as velocidades de operação utilizadas seguiram rigorosamente aquelas utilizadas conforme a praxe da região a fim de se avaliar as condições reais de campo utilizadas. 
Capacidade operacional e eficiência de campo da produção de silagem de milho cultivado em pivô central: estudo de caso

A determinação do tempo foi realizada pela medição do tempo contínuo, ou seja, o tempo gasto para o preparo de toda a área. A velocidade foi determinada cronometrando-se o tempo gasto para o implemento percorrer $50 \mathrm{~m}$ durante o trabalho na área.

A Capacidade Operacional Efetiva foi obtida conforme Equação 1:

$$
\mathrm{COE}=\frac{\mathrm{A}}{\mathrm{t}}(\text { Eq. } 1)
$$

Em que: $\mathrm{COE}=$ capacidade operacional efetiva, ha $\mathrm{h}^{-1} ; \mathrm{A}=$ área trabalhada, ha; $\mathrm{t}=$ tempo trabalhado, $\mathrm{h}$.

A Capacidade Operacional Teórica foi obtida conforme a Equação 2:

$$
\mathrm{COT}=\frac{\mathrm{L} \times \mathrm{v}}{10}(\text { Eq. } 2)
$$

Em que: $\mathrm{COT}=$ capacidade operacional teórica, ha $\mathrm{h}^{-1} ; \mathrm{L}=$ largura de trabalho de cada implemento, $\mathrm{m} ; \mathrm{v}=$ velocidade de trabalho, $\mathrm{km} \mathrm{h}^{-1}$.

De posse dos valores de Capacidade Operacional Teórica e Efetiva a Eficiência foi obtida conforme Equação 3:

$$
\mathrm{EF}=\frac{\mathrm{COE}}{\mathrm{COT}} \times 100(\mathrm{Eq} .3)
$$

Em que: $\mathrm{EF}=$ eficiência, \%.

Todos os valores foram confrontados com o intervalo estipulado pela Norma da ASAE D497.4 (1999).

\section{Resultados e discussão}

Conforme a Tabela 1, a utilização do pulverizador e do distribuidor de Sulfato de Amônio e Cloreto de Potássio, foram as únicas operações que apresentaram eficiências abaixo da indicada pela Norma.

No caso do distribuidor de Sulfato de Amônio e Cloreto de Potássio o tempo para preparo do produto e abastecimento dos tanques influenciaram negativamente o valor da eficiência, já no caso do pulverizador, embora a velocidade utilizada fosse inferior àquela normalizada, o local de abastecimento foi o fator que mais influenciou na baixa eficiência, pois este se localizava distante da área e a calda era preparada no momento do abastecimento. A distância de abastecimento do tanque do pulverizador se localizava a $105 \mathrm{~m}$ da extremidade do pivô, chegando a $275 \mathrm{~m}$ da extremidade oposta.

Estes resultados corroboram àqueles citados por Machado, Queiroz e Reynaldo (2015), ao avaliarem o desempenho operacional de um pulverizador autopropelido de barras, que, o tempo gasto para reabastecimento do reservatório, seguido do tempo de deslocamento para reabastecimento, representaram uma alta influência no tempo de interrupção da aplicação, sendo os mais impactantes.

Os resultados de Capacidade Operacional Efetiva, Teórica e Eficiência de cada operação mecanizada podem

\begin{tabular}{|c|c|c|c|c|c|c|c|c|}
\hline Equipamentos & $\begin{array}{c}\mathrm{L} \\
(\mathrm{m})\end{array}$ & $\begin{array}{c}\mathrm{vr} \\
\left(\mathrm{Km} \mathrm{h}^{-1}\right)\end{array}$ & $\begin{array}{c}v t^{*} \\
\left(\mathrm{Km} \mathrm{h}^{-1}\right)\end{array}$ & $\begin{array}{c}t \\
(h)\end{array}$ & $\begin{array}{c}\mathrm{COE} \\
\left(\mathrm{ha} \mathrm{h}^{-1}\right)\end{array}$ & $\begin{array}{c}\text { COT } \\
\left(h^{\prime} h^{-1}\right)\end{array}$ & $\begin{array}{l}\mathrm{EF} \\
(\%)\end{array}$ & $\begin{array}{c}\text { EFT* }^{*} \\
(\%)\end{array}$ \\
\hline Grade aradora & 1,80 & 4,50 & 7,00 & 4,00 & 0,58 & 0,81 & 70,99 & $70-90$ \\
\hline Grade destorroadora & 2,37 & 6,00 & 7,00 & 4,00 & 1,15 & 1,42 & 80,87 & $70-90$ \\
\hline Semeadora adubadora & 3,00 & 6,00 & 5,00 & 2,50 & 0,92 & 1,80 & 51,11 & $50-75$ \\
\hline Pulverizador com herbicida & 9,00 & 5,00 & 6,50 & 1,30 & 1,77 & 4,50 & 39,32 & $50-80$ \\
\hline Pulverizador com inseticida & 9,00 & 5,00 & 6,50 & 1,30 & 1,77 & 4,50 & 39,32 & $50-80$ \\
\hline $\begin{array}{l}\text { Distribuidor de sulfato de } \\
\text { amônio } \\
\text { e cloreto de potássio }\end{array}$ & 2,80 & 6,00 & 6,50 & 2,00 & 1,15 & 1,68 & 68,45 & $70-90$ \\
\hline Distribuidor de ureia & 2,80 & 6,00 & 6,50 & 3,67 & 1,25 & 1,68 & 74,81 & $70-90$ \\
\hline Colhedora & 0,80 & 3,00 & 5,00 & 18,32 & 0,13 & 0,24 & 52,31 & $50-75$ \\
\hline
\end{tabular}
ser observados na Tabela 1 :

Tabela 1 - Eficiência dos equipamentos utilizados

*Valores estipulados pela Norma ASAE D497.4 MAR99.

Em que: L: largura dos equipamentos; vr: velocidade real; vt: velocidade tabelada pela Norma; t: tempo de trabalho real; COE: Capacidade Operacional Efetiva; COT: Capacidade Operacional Teórica; EF: Eficiência real; EFT: Eficiência tabelada pela Norma. 
Os dados podem ser observados na Figura 01.

Figura 1 - Eficiência dos equipamentos utilizados

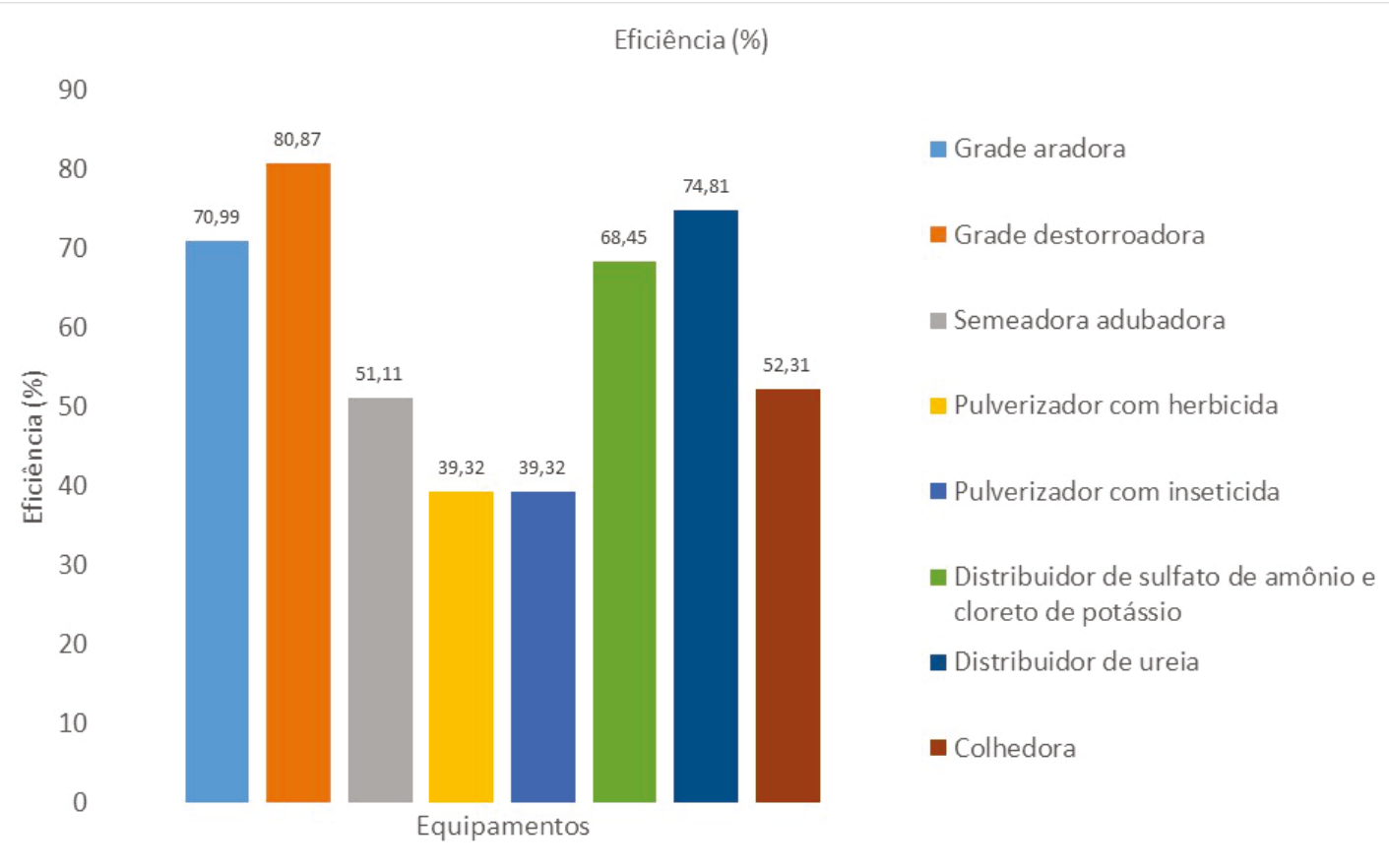

No caso da grade aradora, embora o valor estivesse dentro da faixa estipulada pela Norma, este foi bem baixo, sendo influenciado diretamente pela baixa velocidade, já que este implemento não requer outras paradas que não sejam as de manobra. Recomenda-se a utilização da velocidade mais próxima da estipulada pela Norma. A grade destorroadora apresentou valor satisfatório, influenciado pela velocidade ter se aproximado bastante da Norma.

Farias et al. (2018) avaliando o desempenho operacional e energético de um trator agrícola durante operação de gradagem, com uma grade niveladora de $2,34 \mathrm{~m}$ de largura na velocidade de $6,7 \mathrm{~km} \mathrm{~h}^{-1}$, obtiveram valor de Capacidade de Campo (Operacional) Efetiva de 1,13 ha $^{-1}$, valor este muito próximo do encontrado neste trabalho. Mesmo utilizando uma velocidade abaixo dos autores citados, ou seja, de $6 \mathrm{~km} \mathrm{~h}^{-1}$, encontrou-se, neste caso, o valor de 1,15 ha $^{-1}$, demonstrando alta produtividade e aproveitamento do trabalho efetuado.

A semeadora adubadora, embora também, tenha apresentado valor dentro da faixa estipulada, obteve um valor bem baixo, ou seja, de 51,11\%. Já que a velocidade utilizada foi superior àquela estipulada, o baixo valor pode ser explicado devido também ao tempo de parada para abastecimento de sementes e adubo. Silveira, Yanay e Hurachi (2006), determinando a eficiência de campo de conjuntos de máquinas convencionais, encontraram eficiência média do conjunto trator/semeadora muito próximo de 49,2\%.

Neres et al. (2012), porém, avaliando o desempenho operacional de um trator agrícola e suas implica- ções de uso em alguns atributos físicos de um Latossolo Amarelo, utilizando uma grade aradora, uma grade niveladora e uma semeadora adubadora, concluíram que a semeadora-adubadora obteve maior eficiência do que os demais, valor este diferente dos encontrados neste trabalho. Os autores explicam que este resultado foi devido às condições de umidade do solo, a qual estava em torno de $10 \%$.

O distribuidor de ureia também obteve valor dentro de estipulado pela Norma.

A colhedora de forragem também obteve eficiência dentro da estipulada pela Norma, porém baixo. Ainda que a colheita seja realizada em uma linha apenas, e a velocidade utilizada tenha sido muito baixa, as paradas para troca das carretas contribuíram também para a baixa Eficiência. Já que a troca de carretas se fez necessária, o aumento da velocidade contribuiria para uma maior eficiência.

Além dos fatores explicados anteriormente, o excesso de manobras de cabeceira, já que o formato da área é circular, pode também ter contribuído para diminuição da eficiência de todas as operações.

Os resultados mostraram que o tempo de preparo e abastecimento de insumos, preparo de calda, troca de carreta e o tempo de deslocamento propiciaram menor eficiência às operações no cultivo de milho em pivô central, corroborando os resultados encontrados por Araldi et al. (2013). 
Capacidade operacional e eficiência de campo da produção de silagem de milho cultivado em pivô central: estudo de caso

Simões e Silva (2012) avaliando o desempenho operacional e econômico de um trator agrícola, na operação de irrigação pós-plantio de eucalipto a campo, em distintas áreas de reflorestamento também observaram que, o tempo improdutivo exerceu impacto sobre a capacidade de campo operacional e, consequentemente, aumentou o custo de produção em aproximadamente $30 \%$.

\section{Conclusão}

Os implementos que necessitaram de tempo para preparo e abastecimento de insumos, preparo de calda e troca de carreta foram os que apresentaram menor eficiência;

Mesmo apresentando maior largura do que os outros implementos, o pulverizador apresentou menor eficiência devido ao maior tempo de deslocamento e abastecimento do equipamento;

O tempo total de manobras devido à área circular do terreno pode ter contribuído para a baixa eficiência das operações.

\section{Referências}

Araldi, P. F.; Schlosser, J. F.; Frantz, U. G.; Ribas, R. L.; Santos, P. M. 2013. Eficiência operacional na colheita mecanizada em lavouras de arroz irrigado, Ciência Rural, 43: 445-451. Doi: http://dx.doi. org/10.1590/S0103-84782013000300011.

ASAE (American Society of Agricultural Engineers). 1999. ASAE D497.4 MAR99: Agricultural Machinery Management Data. In: ASAE Standards 2000: Standards, Engineering Practices, and Data. St. Joseph. 350-357.

Baio, F. H. R.; Rodrigues, A. D.; Santos, G. S.; Silva, S. P. 2013. Modelagem matemática para seleção de conjuntos mecanizados agrícolas pelo menor custo operacional, Engenharia Agrícola, 33: 402-410. Doi: http://dx.doi.org/10.1590/S0100-69162013000200018.

Duarte Júnior, J. B.; Garcia, R. F.; Coelho, F. C.; Amim, R. T. 2008. Desempenho de trator-implemento na cana-de-açúcar em sistemas de plantio direto e convencional. Revista Brasileira de Engenharia Agrícola e Ambiental, 12, 653-658. Doi: http://dx.doi.org/10.1590/ S1415-43662008000600013.

Farias, M. S.; Schlosser, J. F.; Martini, A. T.; Bertollo, G. M.; Alvez, J. V. 2018. Desempenho operacional e energético de um trator agrícola durante operação de gradagem. Tecno-Lógica, 22, 213-216. Doi: http:// dx.doi.org/10.17058/tecnolog.v22i2.12125.
Machado, T. M.; Queiroz, D. G. B.; Reynaldo, É. F. 2015. Desempenho operacional de pulverizador autopropelido de barras no município de Sinop - MT. Enciclopédia Biosfera, Centro Científico Conhecer, 11, 875887. Doi: http://dx.doi.org/10.18677/Enciclopedia_Biosfera_2015_128.

Neres, J. S.; Santos, R. S.; Moreira, D. A.; Silva, R. M. 2012. Desempenho operacional de um trator agrícola e suas implicações de uso em alguns atributos físicos de um Latossolo Amarelo em Altamira-PA. Engenharia Ambiental, 9, 62-77.

Pequeno, I. D; Arcoverde, S. N. S.; Cortez, J. W.; Garrido, M. S.; Carvalho, P. G. S. 2012. Desempenho operacional de conjunto trator-grade em argissolo amarelo no semiárido nordestino. Nucleus, 9, 83-92. Doi: http://dx.doi.org/10.3738/nucleus.v9i2.720.

Silveira, G. M.; Yanai, K.; Kurachi, S. A. H. 2006. Determinação da eficiência de campo de conjuntos de máquinas convencionais de preparo do solo, semeadura e cultivo. Revista Brasileira de Engenharia Agrícola e Ambiental, 10, 220-224. Doi: http://dx.doi.org/10.1590/S141543662006000100032 .

Simões, D.; Silva, M. R. 2012. Desempenho operacional e custos de um trator na irrigação pós-plantio de eucalipto em campo. Revista Ceres, 59, 164-170. Doi: http://dx.doi.org/10.1590/S0034-737X2012000200003. 